\title{
Manifestations of the absence of spin diffusion in multipulse NMR experiments on diluted dipolar solids
}

\author{
María Belén Franzoni and Patricia R. Levstein \\ Facultad de Matemática, Astronomía y Física, Universidad Nacional de Córdoba, \\ Ciudad Universitaria, 5000, Córdoba, Argentina
}

\begin{abstract}
Puzzling anomalies previously observed in multipulse NMR experiments in natural abundance ${ }^{29} \mathrm{Si}[\mathrm{A} . \mathrm{E} . \mathrm{Dementyev}, \mathrm{D}$. Li, K. MacLean, and S.E. Barrett, Phys. Rev. B 68, 153302 (2003)] such as long-lived spin echoes and even-odd asymmetries, are also found in polycrystalline $\mathrm{C}_{60}$. Further experiments controlling the phases and tilting angles of the pulse trains, as well as analytical and numerical calculations allowed us to explain the origin of these anomalies. We prove that the observation of long magnetization tails requires two conditions: i) an rf field inhomogeneity able to produce different tilting angles in different sites of the sample and ii) the absence of spin diffusion (non-effective flip-flop interactions). The last requirement is easily satisfied in diluted dipolar solids, where the frequency differences between sites, caused by disorder or other sources, are usually at least one order of magnitude larger than the dipolar couplings. Both conditions lead to the generation of stimulated echoes in Carr-Purcell (CP) and Carr-Purcell-Meiboom-Gill (CPMG) pulse trains. We show, both experimentally and theoretically, that the stimulated echoes interfere constructively or destructively with the normal (Hahn) echoes depending on the alternation or not of the $\pi$ pulse phases in the CP and the CPMG sequences. Constructive interferences occur for the CP and CPMG sequences with and without phase alternation respectively, which are the cases where long magnetization tails are observed. Sequences with two, three and four $\pi$ pulses after the $\pi / 2$ pulse allow us to disentangle the contributions of the different echoes and show how the stimulated echoes originate the even-odd asymmetry observed in both ${ }^{29} \mathrm{Si}$ and $\mathrm{C}_{60}$ polycrystalline samples.
\end{abstract}

During the last years, the control of quantum coherence has become of great importance for quantum information processing and for the development of new nanotechnologies. In the nanoscale, many quantum phenomena become evident and can be exploited. However, interaction with various degrees of freedom of the environment degrades the quantum coherence. As a consequence, it is necessary to understand in detail the mechanisms that produce decoherence and their timescales. Many suggestions to implement quantum computations involve nuclear or electron spins $\stackrel{1.2}{\frac{1}{2}}$ In particular, as silicon has been an essential component of one of the main proposals, $\stackrel{3}{=}$ a group of researchers carried out a series of nuclear magnetic resonance (NMR) measurements to study the spin-spin "decoherence" time $\left(T_{2}\right)$ in ${ }^{29} \mathrm{Si}$ (4.67\% natural abundance, spin $\frac{1}{2}$ ) doped with different concentrations of donors or acceptors $\underline{\underline{4}}$ Their results were completely unexpected and defied explanation to date.

They performed $T_{2}$ measurements with different pulse sequences commonly used with this purpose. They found that it is possible to detect ${ }^{29} \mathrm{Si}$ NMR signals up to much longer times (more than two orders of magnitude) by applying the Carr-Purcell-Meiboom-Gill sequence as compared with the characteristic decay time obtained from the Hahn echo sequence ( $\approx 5.6 \mathrm{~ms}$ ). As these sequences usually give similar results, the difference cannot be disregarded and requires an explanation.

Several tests were performed to determine the origin of this anomalous behavior. First of all, it was evident that there was no dependence on the amount of donors or acceptors in the ${ }^{29} \mathrm{Si}$ sample, even when this could change the linewidth and the spin-lattice relaxation time in one order of magnitude. Moreover, they found the same behavior in a pure single crystal of n.a. ${ }^{29} \mathrm{Si}^{5}$

One hint was that the anomaly seemed to be present in systems with diluted spins. Thus, we implemented a series of related experiments and calculations in a $\mathrm{C}_{60}$ sample. As each molecule has sixty carbon atoms and the magnetic isotope ${ }^{13} \mathrm{C}$, has a natural abundance $1,1 \%$, in a typical sample there are $34.5 \%$ of molecules containing one ${ }^{13} \mathrm{C}, 11.4 \%$ containing exactly two, and $2.4 \%$ containing three.

As a first step, we search for an approximation to $T_{2}$ using the Hahn echo sequence ${ }^{6}\left[H E:\left(\frac{\pi}{2}\right)_{X}-\tau-(\pi)_{Y}-\right.$ $\tau-e c h o]$, where $\tau$ is a variable time. We obtained $T_{2}^{H E} \simeq$ $15 \mathrm{~ms}$. Then, we used different train pulse sequences to measure $T_{2}$, derived from the Carr-Purcell sequence,,$\frac{7}{7}$

$$
\begin{aligned}
& C P 1:\left(\frac{\pi}{2}\right)_{X}-\left[\tau-(\pi)_{X}-\tau-e c h o\right]_{n} \\
& C P 2:\left(\frac{\pi}{2}\right)_{X}-\left[\begin{array}{c}
\tau-(\pi)_{X}-\tau-e c h o-\tau- \\
-(\pi)_{-X}-\tau-e \operatorname{cho}
\end{array}\right]_{n},
\end{aligned}
$$

and the Carr-Purcell-Meiboom-Gill sequence,,$\frac{8}{2}$

$$
\begin{aligned}
& \text { CPMG1: }\left(\frac{\pi}{2}\right)_{X}-\left[\tau-(\pi)_{Y}-\tau-e c h o\right]_{n} \\
& \text { CPMG2: }\left(\frac{\pi}{2}\right)_{X}-\left[\begin{array}{c}
\tau-(\pi)_{Y}-\tau-e c h o-\tau- \\
-(\pi)_{-Y}-\tau-e c h o
\end{array}\right]_{n}
\end{aligned}
$$

Here, the numbers 1 and 2 have been added to the names of the sequences to denote one or two different phases for the $\pi$ pulses.

We found that by applying the $C P M G 1$ sequence or the $C P 2$ sequence, a magnetization tail appears, i.e. the signal remains for times much longer than $T_{2}^{H E}$. Moreover, with both of them we found that by choosing $2 \tau$ $\geqslant T_{2}^{H E}$ the magnetization shows an even-odd behavior as

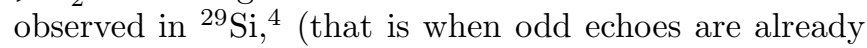
in the noise, even echoes are still visible).

Another unexpected observation was the presence of noticeable stimulated echoes 
when applying pulse sequences of the form, $\left[S E:\left(\frac{\pi}{2}\right)_{X}-\tau-(\pi)_{\varphi_{2}}-t_{1}-(\pi)_{\varphi_{3}}-a c q\right] \quad$ with variable $\tau$ and $t_{1}$. In principle, these sequences involving $\pi$ pulses should not produce stimulated echoes. As we will show, the constructive or destructive interferences between the stimulated and normal echoes enable a phenomenological explanation for the long magnetization tails.

Figure 11 shows the normalized magnetizations acquired by applying the sequences $H E ; C P 1 ; C P 2$; $C P M G 1$ and $C P M G 2$ to polycrystalline $\mathrm{C}_{60}$. In the last four sequences $\tau=1 \mathrm{~ms}$ and signal acquisition is performed at the top of the echo. In all the sequences the durations of the $\pi$ and $\pi / 2$ pulses were carefully set from nutation experiments to $7.0 \mu \mathrm{s}$ and $3.4 \mu \mathrm{s}$, respectively. No effort was done to reduce the rf field inhomogeneity which, for the completely full sample holder, was in the range $15-20 \%$. It is clear from Fig.(11) that the magnetization, measured with the $C P 2$ and $C P M G 1$ sequences, lasts for very long times $(\approx 1 \mathrm{~s})$.

On the other hand, by applying the $C P 1$ or the $C P M G 2$ sequences the decay time is not longer than $T_{2}^{H E}$, but it is remarkable that at short times the magnetization makes a zigzag that, as will become clear, it is also a consequence of the presence of stimulated echoes. Besides, it should be noticed that the magnetization goes through negative values before reaching its final asymptotic zero value.

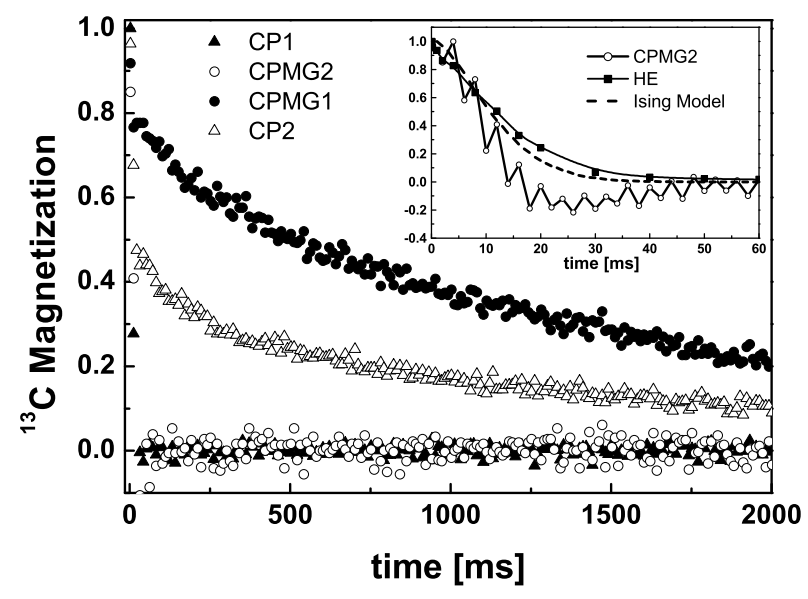

FIG. 1: Comparison of the ${ }^{13} \mathrm{C}$ magnetizations acquired with different $T_{2}$ sequences $(\tau=1 \mathrm{~ms})$. The insert shows the decay observed with the Hahn echo, the decay simulated by considering only the Ising terms of the dipolar interaction and the short time regime of the $C P M G 2$ sequence.

The first question was if the $T_{2}^{H E}$ measured for the $\mathrm{C}_{60}$ sample could be directly assigned to the dipolar interaction within the ${ }^{13} \mathrm{C}$ nuclei. Our measurements of the spin-lattice relaxation time yielded $T_{1} \simeq 35$ s reflecting that there are no efficient mechanisms for this kind of relaxation that should be taken into account in our analysis of the "decoherence" time. Thus, the main interactions in this system led us to write the Hamiltonian in the rotating frame as

$\mathcal{H}=\sum_{i=1}^{N}\left[\hbar \delta \omega_{i} I_{i}^{z}+\sum_{j>i}^{N}\left(a_{i j} I_{i}^{z} I_{j}^{z}+b_{i j}\left(I_{i}^{+} I_{j}^{-}+I_{i}^{-} I_{j}^{+}\right)\right)\right]$,

where $\delta \omega_{i}$ represents the chemical shift for spin $i$ (relative to on-resonance spins); $a_{i j}=\left(\gamma^{2} \hbar^{2} / r_{i j}^{3}\right)\left(1-3 \cos ^{2} \theta_{i j}\right)$; $b_{i j}=-a_{i j} / 4$ with $\theta_{i j}$ the angle between the internuclear vector $\mathbf{r}_{i j}$ and the static magnetic field. The gyromagnetic ratio of ${ }^{13} \mathrm{C}$ is $\gamma=6.7283 \times 10^{7} \mathrm{rad} \mathrm{T} \mathrm{T}^{-1} \mathrm{~s}^{-1}$ and the center to center distance between neighboring molecules is $r_{i j}=10 \AA .9$ It should be noticed that because of the rapid isotropic rotations of the buckyballs in the solid, the spins can be considered to lie at the center of the molecule 10

The Lorentzian full width at half maximum, $F W H M \simeq 167 \mathrm{~Hz}$, corresponding to a free induction decay time $T_{2}^{*} \simeq 1.91 \mathrm{~ms}$ is almost an order of magnitude shorter than $T_{2}^{H E} \simeq 15.0 \mathrm{~ms}$, thus reflecting the sample inhomogeneity. Then, it is usual to make the unlike spins approximation $\left|a_{i j}\right| \ll\left|\delta \omega_{i}-\delta \omega_{j}\right|$, and truncate the $b_{i j}$ terms, leaving only the Ising contribution. Under these conditions, one can use the product operator formalism, 11 to obtain an analytical expression for the magnetization. Starting from the initial equilibrium density operator in the conventional strong field and high temperature approximations:

$$
\rho(t=0) \propto \sum_{i} I_{i}^{z}
$$

the application of the Hahn echo sequence with variable inter-pulse time $\tau=k \tau$, leads to the expression

$$
\left\langle I_{y}(k 2 \tau)\right\rangle=\sum_{i}^{N} I_{i}^{y}(0)\left\{\prod_{j>i}^{N} \cos \left(a_{i j} k \tau\right)\right\} .
$$

Our calculation for a polycrystalline sample of $\mathrm{C}_{60}$ involves a set of 600 pieces $(\approx 4000$ unit cells each $)$ with different representative orientations with respect to the external magnetic field and a random distribution of molecules with one, two or three spins each considering the proportions expressed above. The characteristic decay time of the analytic curve is in very good agreement with the experimental one obtained from the Hahn echo sequence (see Ising model in Fig [1), manifesting that $T_{2}^{H E}$ can be directly associated with the dipolar interaction.

In order to look for the even-odd behavior reported for the $\mathrm{Si}$ sample $e^{4}$ in $\mathrm{C}_{60}$, we took long inter-pulse separations $\left(2 \tau>T_{2}^{H E}\right)$. We observed the anomalous behavior 


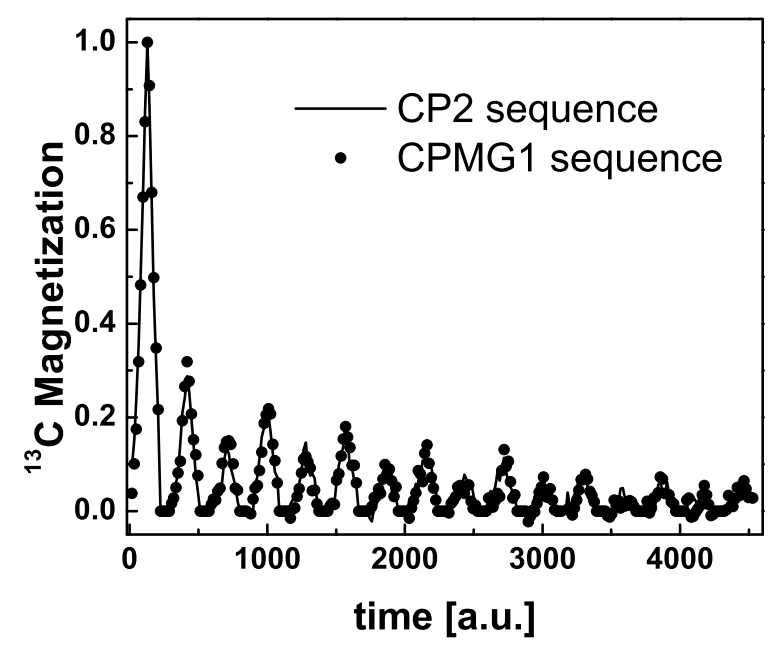

FIG. 2: Even-odd effect observed with the $C P M G 1$ and $C P 2$ sequences in a $\mathrm{C}_{60}$ polycrystalline sample. The interpulse separation is $\tau=8 \mathrm{~ms}$.

by applying the $C P M G 1$ and the $C P 2$ sequences, as displayed in Fig 2 for $\tau=8 \mathrm{~ms}$. While the first echo, occurring at $t=16 \mathrm{~ms}$ is higher than the second one, occurring at $t=32 \mathrm{~ms}$, the third is lower than the forth, and so on. Moreover, the same sequence with $\tau=25 \mathrm{~ms}$ produces a first echo which is smaller than the second one.

So far, all the results reported in silicon were also manifested in the $\mathrm{C}_{60}$ sample, but there was still one more thing to check, the presence of stimulated echoes.

We applied a sequence of three pulses $\underline{\underline{\underline{6}}}$

$$
S E:\left(\frac{\pi}{2}\right)_{\varphi_{1}}-\tau-(\pi)_{\varphi_{2}}-t_{1}-(\pi)_{\varphi_{3}}-a c q,
$$

taking $\varphi_{1}=X$ in all the experiments and varying $\varphi_{2}$ and $\varphi_{3}$ in correspondence with the sequences we used in the first experiments. For these stimulated echo sequences, we denote $\left[C P M G 1^{S E}: \varphi_{2}=Y ; \varphi_{3}=Y\right]$, $\left[C P 1^{S E}: \varphi_{2}=X ; \varphi_{3}=X\right]$

$\left[C P M G 2^{S E}: \varphi_{2}=Y ; \varphi_{3}=-Y\right], \quad$ and $\left[C P 2^{S E}: \varphi_{2}=X ; \varphi_{3}=-X\right]$. At a time $t_{1}-\tau$ after the third pulse, the normal echo (i.e. the refocusing of the Hahn echo at time $\tau$ after the second pulse) appeared, and for all the sequences a stimulated echo peaked at time $\tau$ after the third pulse.

A very remarkable fact is that by applying the $C P M G 1^{S E}$ or the $C P 2^{S E}$ sequence, the stimulated and normal echoes have the same phases, but when applying the $C P M G 2^{S E}$ or the $C P 1^{S E}$ sequences they have opposite phases, as shown in Fig 3 for the $C P M G$ variations. Using the superoperator formalism, $, 12,13$
for an arbitrary three-pulse sequence

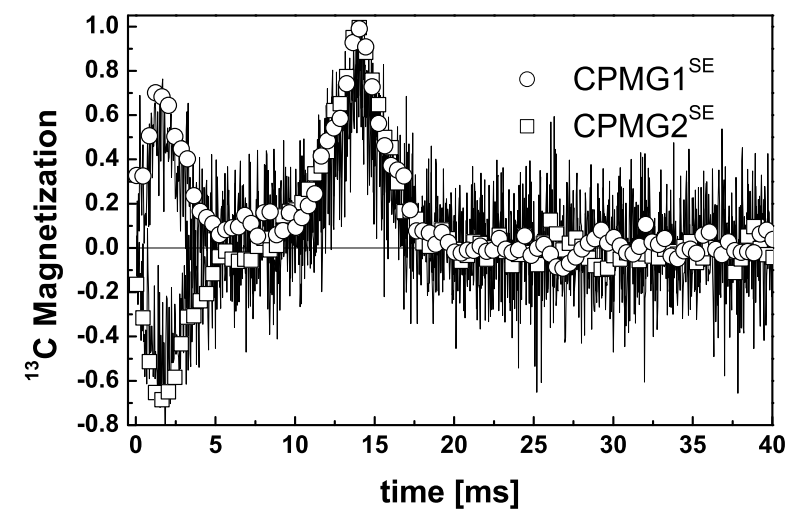

FIG. 3: Stimulated echoes observed with the $C P M G 1^{S E}$ and $C P M G 2^{S E}$ sequences in a $\mathrm{C}_{60}$ polycrystalline sample. The parameters are $\tau=1 \mathrm{~ms}$ and $t_{1}=15 \mathrm{~ms}$, giving rise to a stimulated and a normal echo at $1 \mathrm{~ms}$ and $14 \mathrm{~ms}$ after the last pulse, respectively. The circles and squares represent splines (average of 20 original datapoints per symbol) performed to guide the eye. Notice the inversion of the phase in the stimulated echo.

$\left[\left(\beta_{1}\right)_{\varphi_{1}}-\tau-\left(\beta_{2}\right)_{\varphi_{2}}-t_{1}-\left(\beta_{3}\right)_{\varphi_{3}}-a c q\right]$ where $\beta_{i}$ and $\varphi_{i}$ are arbitrary tilting angles and phases in the $X Y$ plane respectively, we analytically solved the dynamical equations in the delta-pulse approximation. By taking into account that the FWHM $\simeq 167$ $\mathrm{Hz}$ and $\left(\pi T_{2}^{H E}\right)^{-1} \simeq 21.2 \mathrm{~Hz}$, we can neglect the spin-spin interactions and work with the Hamiltonian $\mathcal{H}=\hbar \sum_{i=1}^{N} \delta \omega_{i} I_{i}^{z}$,

and the initial condition given in Eq.(4). Under these approximations, the following response function is obtained

$$
\begin{aligned}
& G\left(t>\tau+t_{1}\right)=\quad e^{i \varphi_{1}} f_{1} G(t) \quad+e^{i\left(2 \varphi_{3}-2 \varphi_{2}+\varphi_{1}\right)} f_{2} G\left(t-2 t_{1}\right) \\
& +e^{i\left(\pi+2 \varphi_{2}-\varphi_{1}\right)} \quad f_{3} G(t-2 \tau) \\
& +e^{i\left(\pi+2 \varphi_{3}-\varphi_{1}\right)} \quad f_{4} G\left(t-2 t_{1}-2 \tau\right) \\
& +e^{i\left(\pi+\varphi_{3}-\varphi_{2}+\varphi_{1}\right)} f_{5} G\left(t-t_{1}\right) \\
& +e^{i\left(\pi+\varphi_{3}+\varphi_{2}-\varphi_{1}\right)} f_{6} G\left(t-t_{1}-2 \tau\right) \\
& +e^{i\left(\pi+2 \varphi_{3}-\varphi_{2}\right)} \quad f_{7} G\left(t-2 t_{1}-\tau\right),
\end{aligned}
$$

where $G(t)=\frac{1}{N} \sum_{j=1}^{N} \exp \left(i t \delta \omega_{j}\right)$ represents the normalized FID, the time $t$ is the total time starting at the very first pulse, and the signal amplitudes $f_{j} \equiv f_{j}\left(\beta_{1}, \beta_{2}, \beta_{3}\right)$ are determined from the exact calculation: 


$$
\begin{aligned}
& f_{1}=\sin \beta_{1} \cos ^{2} \frac{\beta_{2}}{2} \cos ^{2} \frac{\beta_{3}}{2} \\
& f_{2}=\sin \beta_{1} \sin ^{2} \frac{\beta_{2}}{2} \sin ^{2} \frac{\beta_{3}}{2} \\
& f_{3}=\sin \beta_{1} \cos ^{2} \frac{\beta_{3}}{2} \sin ^{2} \frac{\beta_{2}}{2} \\
& f_{4}=\sin \beta_{1} \cos ^{2} \frac{\beta_{2}}{2} \sin ^{2} \frac{\beta_{3}}{2} \\
& f_{5}=\frac{1}{2} \sin \beta_{1} \sin \beta_{2} \sin \beta_{3} \\
& f_{6}=\frac{1}{2} \sin \beta_{1} \sin \beta_{2} \sin \beta_{3} \\
& f_{7}=\cos \beta_{1} \sin \beta_{2} \sin ^{2} \frac{\beta_{3}}{2}
\end{aligned}
$$

The stimulated echo signal is the one that peaks at total time $t=t_{1}+2 \tau$. Its amplitude is given by the expression $f_{6}$, evidencing that for sequences like the ones applied here (i.e. $\beta_{2}=\beta_{3}=\pi$ ), it cancels out. Consequently, the stimulated echo should not appear.

The normal echo, second term in Eq.(7), peaks at a total time $t=2 t_{1}$. Its phase in the $C P M G 1\left(\varphi_{1}=0\right.$, $\left.\varphi_{2}=\pi / 2, \varphi_{3}=\pi / 2\right)$ or in the CP2 $\left(\varphi_{1}=0, \varphi_{2}=0\right.$, $\left.\varphi_{3}=\pi\right)$ sequence is $e^{i\left(2 \varphi_{3}-2 \varphi_{2}+\varphi_{1}\right)}=1$ coinciding with that of the stimulated echo $e^{i\left(\pi+\varphi_{3}+\varphi_{2}-\varphi_{1}\right)}=1$. However, if we apply the CPMG2 $\left(\varphi_{1}=0, \varphi_{2}=\pi / 2, \varphi_{3}=-\pi / 2\right)$ or the $C P 1\left(\varphi_{1}=0, \varphi_{2}=0, \varphi_{3}=0\right)$ sequences they are in opposite phases, because the phase of the stimulated echo is inverted. Here, we have used $\varphi_{i}=0$ for the $X$ axis and it should be noticed that under the experimental conditions $\left(\beta_{2}=\beta_{3}\right) f_{2}$ and $f_{6}$ are always positive. Then, the remarkable fact is that these analytical calculations agree with the experimental result for any pair of tilting angles $\beta_{2}=\beta_{3} \neq \pi$. This lead us to conclude that not all the spins are affected by $\pi$ pulses. We performed the three-pulse experiment for $\varphi_{1}=0, \varphi_{2}=\pi / 2, \varphi_{3}=\pi / 2$, $\beta_{1}=\pi / 2$ and $\beta_{2}=\beta_{3}$ varying in the range $[0,5 \pi / 2]$ with $\tau=14 \mathrm{~ms}$ and $t_{1}=15 \mathrm{~ms}$. While the experiments reproduce the functional form of $f_{6}$, there are no zeroes in the magnitude of the stimulated echoes for any value of $\beta_{2}$. It is noticeable that the minimum magnitude of the SE, obtained for $\beta_{2}=\beta_{3} \approx \pi$ is approximately $30 \%$ of the maximum value that occurs for $\beta_{2}=\beta_{3}=\pi / 2$ (data not shown).

It is evident that if we take $t_{1}=2 \tau$ in the three-pulse sequences (6) the normal and the stimulated echoes will appear at the same time, and as can be seeing in fig (3), they will interfere constructively in the $C P M G 1$ and the $C P 2$ sequences or destructively in the $C P M G 2$ and the $C P 1$ ones. As a consequence of these constructive interferences and the well known fact that stimulated echoes have much longer decay times, 6.12 the magnetization tails appear.

In order to analyze the different contributions to the even-odd asymmetry in the echoes, displayed in Fig 2 we performed experiments with two, three and four $\pi$ pulses

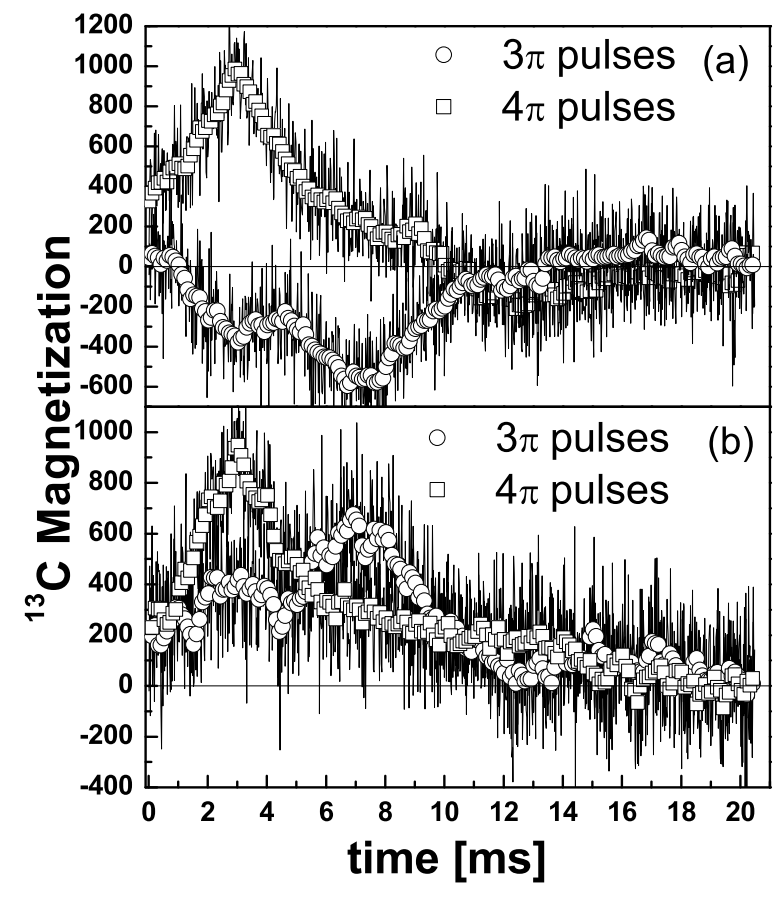

FIG. 4: Echoes obtained after three and four $\pi$ pulses with the (a) $C P 2^{S E}$ and (b) $C P M G 1^{S E}$-like sequences. The time between the $\pi / 2$ and the $\pi$ pulse is $\tau=3 \mathrm{~ms}$, while the intervals between $\pi$ pulses are $t_{1}=10 \mathrm{~ms}$. For three $\pi$ pulses the stimulated echo appears at $t_{1}-\tau=7 \mathrm{~ms}$ after the last pulse, while the normal echo appears at $\tau$. The normal and stimulated echo times after the last $\pi$ pulse are exchanged in the case of four $\pi$ pulses. Notice that after four $\pi$ pulses, the normal echoes have almost dissapeared. The circles and squares represent splines (20 original datapoints averaged).

after the $\pi / 2$ pulse with interpulse separations precluding the overlap of the normal and stimulated echoes. In particular, we set the separation between the $\pi / 2$ and the $\pi$ pulses $\tau=3 \mathrm{~ms}$, while the separations between $\pi$ pulses where $t_{1}=10 \mathrm{~ms}$. The results for three and four $\pi$ pulses for the case of a $C P 2^{S E}$ and $C P M G 1^{S E}$-like sequences are shown in Fig. 目 (a) and (b) respectively.

As can be seen, the stimulated echo occurring after four $\pi$ pulses, i.e. at total time $t=3 t_{1}+2 \tau$ or $\tau$ after the last pulse, is larger than the one after three $\pi$ pulses $t=3 t_{1}$ (or $t_{1}-\tau$ after the last pulse) in both cases. We use the same formalism and Hamiltonian applied for the three-pulse sequences, setting a single tilting angle $\beta$ for all the pulses after the $\pi / 2$ one, with $\varphi_{1}=0$ and $\varphi_{i}=0$ or $\pi$ alternatively for $i \neq 1\left(C P 2^{S E}\right)$ or $\varphi_{1}=0$ and $\varphi_{i}=\pi / 2$ for $i \neq 1\left(C P M G 1^{S E}\right)$. Thus, we obtained for the stimulated echo amplitudes the functions: $A_{4 \pi}=1 / 2 \sin ^{2} \beta\left(5 / 2 \cos ^{2} \beta-\cos \beta+1 / 2\right)$ and $A_{3 \pi}=\mp 1 / 2 \sin ^{2} \beta(1-\cos \beta)$. The minus sign in $A_{3 \pi}$ corresponds to the $C P 2^{S E}$ sequence. Again, it is evident 
that for $\beta=\pi$, there should be no stimulated echo, i.e. $A_{4 \pi}=A_{3 \pi}=0$. However, it can be seen from these formulae that for angles in the interval $\beta=\pi(1 \pm 0.35)$, one gets $A_{4 \pi}>A_{3 \pi}$, which is in qualitative agreement with the experimental observation. Numerical calculations for longer sequences (i.e. a larger number of $\pi$ pulses) support this alternation of amplitudes. Consequently, having proved that the stimulated echoes interfere constructively with the normal echoes in the sequences $C P M G 1$ and $C P 2$, we have found the explanation for the evenodd asymmetry observed in Fig 2

In order to further verify the fact that not all the sites in the sample are experiencing the same externally fixed tilting angle, we applied the same sequences (11) and (2) but applying our best $2 \pi$ or $4 \pi$ pulses (zeroes in the nutation experiment), instead of $\pi$ pulses. In all of them, we kept the first $\left(\frac{\pi}{2}\right)_{X}$ pulse. Under these conditions, if the pulses were perfect, one would expect to acquire just an FID signal. This is exactly what we got by applying the $C P M G 2_{(2 \pi)}$ or the $C P 1_{(2 \pi)}$ sequences or their equivalent with $4 \pi$ pulses. Instead, by applying the $C P M G 1_{(2 \pi)}$ or the $C P 2_{(2 \pi)}$ sequences the magnetization tails reappeared as also occurred with the $4 \pi$ pulses.

The conclusion that not all the spins experience the same tilting pulse should not be surprising, as it is well known that the rf field inhomogeneity may be around 5$10 \%$ in ordinary experimental conditions. However, the remaining question is why these long tails do not appear when working with magnetically concentrated samples as usually occurs in solid-state ${ }^{1} \mathrm{H}-\mathrm{NMR}$ measurements. While the rf field inhomogeneities occur in all cases, in diluted systems, the dynamical contribution of the dipolar interaction (i.e. the flip-flop part) is not effective. Then, it can be conceived that in concentrated systems, there are different tilting angles in different sites, but during the interpulse time the defects or excesses in the $\pi$ pulses are exchanged among the sites because of the flip-flop, thus, effectively averaging to the externally fixed pulse. By contrast, in diluted systems the imperfection over one site has always the same sign or direction, originating the stimulated echoes and consequently the long tails. This fact was verified through numerical simulations where imperfections up to $10 \%$ in the " $\pi$ pulses" were randomly applied to different spin sites. But a very important ingredient in this explanation is that the disorder in energies among the different sites must be greater than the dipolar couplings. This condition occurs quite naturally in diluted systems because the dipolar interaction is very small; thus, any interaction capable to produce differences in site energies will be enough to hinder the flip-flop dynamics. As a practical warning, one should consider the fact that magnetically diluted samples require larger amounts making more serious the problem of $\mathrm{rf}$ field inhomogeneity.

The long tails obtained in our experiments can not be interpreted as a decrease in the decoherence rate of the spin system. Because of the pulse imperfections, some magnetization is preserved in the direction of the external magnetic field, having a decay time in the order of the spin-lattice relaxation time ${ }^{12}$

Our findings may be significant for qubit coherence control in a nuclear spin bath, as occurs with electron spins in a lattice containing $\mathrm{Si}, \mathrm{GaAs}$, In or $\mathrm{Al}^{15.16} \mathrm{In}$ these situations, the electron spin coherence is limited by spectral diffusion arising from dipolar fluctuations of lattice nuclear spins.

Another important aspect to discuss is that, as it is well known, the dipolar dynamics is reversible, $\stackrel{17,18,19}{1}$ and there is no real loss of information in the collective spin system because of it. In this sense, a more relevant measurement of "decoherence time" in $\mathrm{C}_{60}$, would involve an homonuclear decoupling as performed by Ladd et al. ${ }^{14}$ in silicon. Under those conditions, more detailed experiments are required to test if there is still a contribution from stimulated echoes. We find no reason to discard it beforehand, because the necessary conditions: rf inhomogeneity and no flip-flop dynamics will still be present.

The absence of nuclear spin diffusion observed in our experiments and in those performed in n.a. ${ }^{29} \mathrm{Si}, \frac{4}{,}$ seems to be a particular manifestation of the localization phenomenon ${ }^{20.21}$ However, as one is working with a $3-\mathrm{d}$ many-body system, the complexity of a theoretical approach is beyond the scope of this work. Nevertheless, NMR studies as a function of nuclear spin concentrations and using different time windows may help to search for a possible phase transition, opening up a new way to look at localization phenomena.

We acknowledge support from Fundación Antorchas, CONICET, FoNCyT, and SeCyT-UNC. P.R.L. is a member of the Research Career and M.B.F a doctoral fellow of CONICET. We benefitted from fruitful discussions with H.M. Pastawski on localization phenomena.
1 D. P. DiVincenzo, Science 270, 255 (1995).

2 D. G. Cory, A. F. Fahmy, and T. F. Havel, Proc. Natl. Acad. Sci. U.S.A. 94, 1634 (1997).

3 B. Kane, Nature 393, 133 (1998).

4 A. E. Dementyev, D. Li, K. MacLean, and S. E. Barrett, Physical Review B 68, 153302 (2003).

5 R. Ramos, A. E. Dementyev, D. Li, K. MacLean, Y. Dong, and S. E. Barrett, Communication at the APS March Meeting p. Y36.10 (2004).

6 E. L. Hahn, Phys. Rev. 80, 580 (1950).

7 H. Y. Carr and E. M. Purcell, Phys. Rev. 94, 630 (1954).

8 E. Fukushima and S. Roeder, Experimental pulse NMR, A nuts and bolts approach (Addison Wesley, 1981).

9 P. A. Heiney, J. E. Fischer, A. R. McGhie, W. J. Romanow, 
A. M. Denenstein, J. J. P. McCauley, and A. B. Smith, Phys. Rev. Lett. 66, 2911 (1991).

10 R. Tycko, G. Dabbagh, R. Flemming, R.C.Haddon, A. Makhija, and S. Zahurak, Phys. Rev. Lett. 67 (1991).

11 C. Slichter, Principles of magnetic resonance (SpringerVerlag, 1990), 3rd ed.

12 M. Mehring and V. A. Weberruß, Object-Oriented Magnetic Resonance (Academic Press, 2001).

13 R. R. Ernst, G. Bodenhausen, and A. Wokaun, Principles of Nuclear Magnetic Resonance in One and Two Dimensions (Oxford Science Publications, 1988).

14 T. D. Ladd, D. Maryenko, Y. Yamamoto, E. Abe, and K. M. Itoh, Phys. Rev. B 71, 014401 (2005).

15 R. de Sousa, N. Shenvi, and K. B. Whaley, Phys. Rev. B
72, 045330 (2005).

16 E. Abe, K. M. Itoh, J. Isoya, and S. Yamasaki, Phys. Rev. B 70, 033204 (2004).

17 W. K. Rhim, A. Pines, and J. S. Waugh, Phys. Rev. Lett. 25, 218 (1970).

18 S. Zhang, B. H. Meier, and R. R. Ernst, Phys. Rev. Lett. 69, 2149 (1992).

19 P. R. Levstein, G. Usaj, and H. M. Pastawski, J. Chem. Phys. 108, 2718 (1998).

20 P. W. Anderson, Phys. Rev. 109, 1492 (1958).

21 P. W. Anderson, Reviews of Modern Physics 50, 191 (1978) 